Diabetologe 2012 $\cdot 8: 94-97$

DOI 10.1007/s11428-011-0769-x

(c) Springer-Verlag 2012

\section{W.A. Scherbaum}

Klinik für Endokrinologie, Diabetologie und Rheumatologie,

European Training Centre in Endocrinology and Metabolism, Universitätsklinikum Düsseldorf

\section{Individualisierte Medizin in der Diabetologie}

krepanz zwischen den formulierten evidenzbasierten Leitlinien der Diabetologie und den Anwendungsszenarien bei individuellen Patienten.

Wie kann es möglich sein, die Implementierung von Leitlinien streng an den evidenzbasierten Leitlinien (EBL) auszurichten, bei denen prospektive, multizentrische, placebokontrollierte Studien und entsprechende Metaanalysen derselben den obersten Stellenwert einnehmen? Wir alle wissen, dass dies entweder nicht die Patienten sind, die wir in der Praxis wirklich sehen, oder dass oft vielen sehr unterschiedlichen Patienten in einer Studie ein einheitliches Behandlungsschema übergestülpt wird, um die Ergebnisse statistisch besser auswerten zu können. Nein, unsere echten Patienten erkennen wir meist nur in sehr kleinen Studien wieder.

\section{> Die eigentlichen Behandlungsziele bei Diabetespatienten gehen weit über die Frage der Blutglukose oder des $\mathrm{HbA}_{1 \mathrm{c}}$-Werts hinaus.}

Es geht nicht nur um diese und viele andere Surrogatparameter wie Blutdruck, Cho- lesterin, Körpergewicht, sondern auch um die Kompatibilität der Therapie mit der Situation des Patienten, Vermeidung schädlicher Nebenwirkungen der Behandlung und um die Gesamtprognose.

\section{Risikoevaluation zur \\ Bestimmung der Therapieziele - Rolle von Risikorechnern}

Für die bessere Bestimmung der Therapieziele ist es hilfreich, die Gesamtprognose quo ad vitam zu kennen. Aus der Sichtweise eines einzigen Fachs und einzelner Parameter ist es kaum möglich, die Prognose eines Patienten realistisch abzuschätzen. Bei der Evaluation des Gesamtrisikos können Risiko-Scores eingesetzt werden, die aus den Daten großer klinischer „Outcome“-Studien wie z.B. der United Kingdom Prospective Diabetes Study (UKPDS Risk Engine), den Daten der Framingham-Studie (Framingham Risk Score) oder der Prospective Cardiovascular Münster Study (Prospective Cardiovascular Münster Score) generiert wurden und erlauben, mit „risk engines“ $z$.B. aus den Angaben über Alter, Geche Zentrum für Qualität in der Medizin (ÄZQ) ist bei der Entwicklung von Diabetesleitlinien von besonderer Bedeutung, weil an der Diabetesbehandlung ganz verschiedene Fachspezialisten und insbesondere auch die Hausärzte beteiligt sind (Nationale VersorgungsLeitlinien, www. aezq.de, [4]). Dies kommt in der Formulierung der übergeordneten Therapieziele der Nationalen Versorgungsleitlinie Diabetes zum Ausdruck (• Abb. 1).

\section{Dilemma der Leitlinien}

Was in der Diabetologie erst jetzt so richtig klar wird, ist die teilweise krasse Dis-
Abb. $1>$ Behandlungsalgorithmus zur Festlegung der Therapieziele beim Typ-2Diabetes

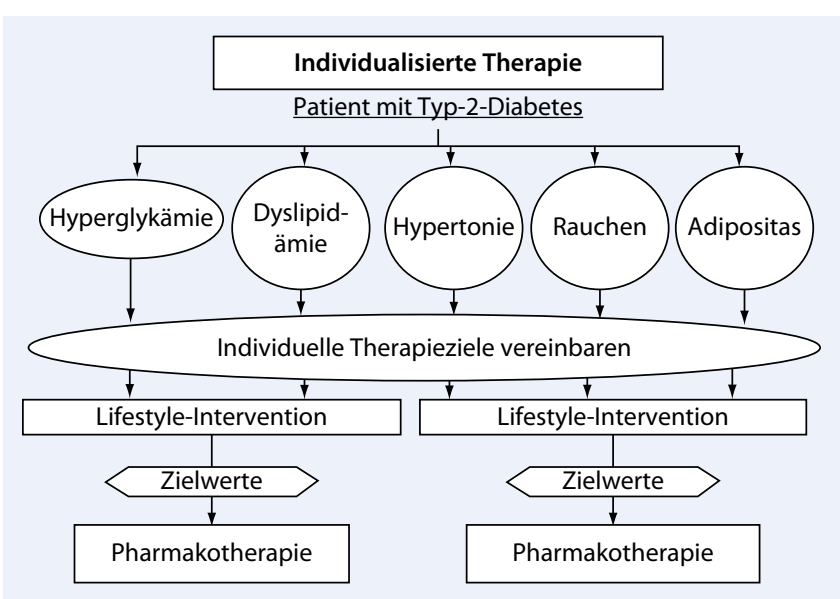


schlecht, Dauer des Diabetes, Vorerkrankungen, $\mathrm{HbA}_{1 \mathrm{c}}$, Blutdruck, Gesamtcholesterin, „High-density-lipoprotein“(HDL)Cholesterin, Albuminurie, Raucherstatus u. a. das individuelle kardiovaskuläre Risiko zu berechnen. Solche schnell verfügbaren Daten sind auch für das Patientengespräch sehr nützlich, weil sich damit u. a. die Möglichkeiten für eine Risikoreduktion bei verschiedenen therapeutischen Optionen zeigen lässt, was zur Motivation des Patienten und zur Therapietreue beiträgt. Zum Beispiel kann damit sehr leicht anschaulich gemacht werden, welchen Vorteil es für die Überlebenswahrscheinlichkeit bringt, wenn eine Mikroalbuminurie oder die Progression zum Nierenversagen verhindert werden kann [1].

\section{Unterschiedliche Therapieziele bei verschiedenen Patientengruppen}

Die Liste der in diesem Heft bearbeiteten Patientengruppen gibt einen Eindruck davon, wie heterogen sich die Patienten in der Diabetologie darstellen - und entsprechend unterschiedlich sind die jeweiligen Therapieziele sowie die erforderlichen individuellen Therapieansätze. Matthaei und Thienel stellen die Therapieziele und Behandlungsempfehlungen bei sonst gesunden Patienten mit Typ-2-Diabetes im mittleren Erwachsenenalter dar, die als allgemeine Ausgangsbasis für die Beschreibungen von abweichenden Therapiezielen und Behandlungsmaßnahmen gelten kann. Schon bei der Lektüre der Liste der Themen dieses Hefts wird

\section{Hier steht eine Anzeige.}

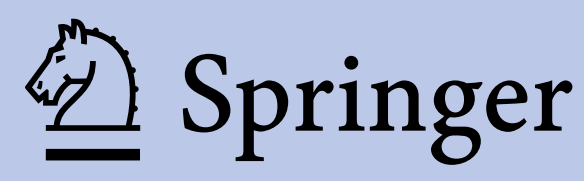




\section{Infobox 1 Liste der wichtigsten speziellen Aspekte und Patientengruppen}

- Sonst Gesunde im jungen und mittleren Erwachsenenalter $^{\mathrm{a}}$

- Kinder und Jugendliche ${ }^{\mathrm{a}}$

- Schwangerschaft ${ }^{\mathrm{a}}$

- Sozialer Status, Beruf, z. B. Kraftfahrer, Pilot

- Adipositas, insbesondere extreme Adipositas $^{\mathrm{a}}$

- Komedikation, Unverträglichkeit von Medikamenten

- Mikro- und makrovaskuläre Langzeitfolgen des Diabetes

- Nephropathie, insbesondere Dialyse

- Retinopathie und Makulopathie, insbesondere Sehstörung/Blindheit ${ }^{\mathrm{a}}$

- Neuropathie, insbesondere schwere Formen

- Insbesondere autonome Neuropathie mit Gastroparese

- Schmerzhafte Polyneuropathie

- Polyneuropathie mit schweren motorischen Einschränkungen

- Diabetisches Fußsyndrom

- KoronareHerzkrankheitundHerzinsuffizienz, speziell Herzinfarkt ${ }^{\mathrm{a}}$

- ZerebrovaskuläreSchäden,insbesondere Schlaganfall

- Geriatrische Patienten ${ }^{\mathrm{a}}$

- "Go-go": mobil, geistig und motorischfunktionell guter Zustand

- "Slow-go":gehandicapt,motorisch-funktionell eingeschränkt

- "No-go":immobil,motorisch-funktionell extrem eingeschränkt

- Schwere Grunderkrankungen mit stark eingeschränkter Lebenserwartung ${ }^{a}$

- Patienten mit kognitiven Einschränkungen, insbesondere Demenz ${ }^{\mathrm{a}}$

- Patienten mit Depressionen ${ }^{a}$

- Patienten mit glukokortikoidinduziertem Diabetes $^{\mathrm{a}}$

- Diabetes bei Mukoviszidose

- Formen von „maturity-onset diabetes of the young" (MODY)

- Diabetespatienten im Krankenhaus ${ }^{\mathrm{a}}$

- Krankenhausaufnahmewegen diabetischer Entgleisung, z. B. Koma

- Aufnahme zur nichtdiabetologischen Diagnostik oder Therapie

- Perioperative Situation

a Diese Patientengruppen werden im vorliegenden Heft von Der Diabetologe besprochen. ersichtlich, dass diese Abweichungen der Behandlungsziele in verschiedenen Gruppen sehr groß sein müssen:

- Kleinkinder, Kinder und Jugendliche (Kies, Leipzig),

- Diabetes bei Betagten

(Wernecke, Hamburg; Bahrmann, Erlangen; Zeyfang, Stuttgart),

- multimorbide Menschen mit eingeschränkter Lebenserwartung (Schumm-Draeger, München),

- Dialysepatienten (Kleophas, Düsseldorf),

- Patienten mit glukokortikosteroidinduziertem Diabetes (Lobnig, Düsseldorf),

- Patienten mit ausgeprägter Adipositas (Hauner, München),

- Diabetespatienten mit koronarer Herzkrankheit (KHK) und Herzinsuffizienz

(Hess u. Marx, Aachen),

- Patienten mit Diabetes und Demenz (Lange, Hannover),

- Patienten mit Diabetes und Depression (Kruse, Gießen, u. Kulzer, Bad Mergentheim)

- Schwangere mit Diabetes (Kleinwechter, Kiel),

- Patienten mit proliferativer diabetischer Retinopathie und Blinde (Lemmen, Düsseldorf) sowie

- Diabetespatienten im Krankenhaus (Siegel, Limburg).

\section{Subgruppen von Patienten mit speziellen Therapieanforderungen}

Selbst innerhalb der oben genannten Gruppen von Diabetespatienten geht es darum, Subgruppen zu definieren, für die spezielle Therapieanforderungen und Therapieziele gelten. Ein gutes Beispiel sind die geriatrischen Patienten, bei denen die Schere der körperlichen und der geistigen Leistungsfähigkeit weit auseinandergeht. Hier haben die geriatrisch tätigen Diabetologen die anschauliche Gruppierung in "go-go", „slow-go" und "no-go" eingeführt, um die unterschiedlichen Vorgaben für die Therapie sichtbar zu machen und besser zu definieren. Oder die Patienten mit Diabetes im Krankenhaus, von denen nur der kleinste Teil zur besseren Einstellung des Diabetes aufgenommen wurde. Bei dem weitaus größten Teil der Diabetespatienten im Krankenhaus stehen andere Probleme im Vordergrund, wie z. B. Spätschäden des Diabetes an Augen, Nieren, Herz und Gefäßen, ein diabetisches Fußsyndrom, andere akute oder chronische Erkrankungen, bei denen der Diabetes eine sog. Nebendiagnose darstellt, Diabetespatienten, die nur kurzfristig wegen kleinerer diagnostischer oder therapeutischer Prozeduren stationär aufgenommen wurden, oder auch Diabetespatienten, bei denen eine Operation durchgeführt wird. Hierbei ist es besonders wichtig, von dem Diabetes $\mathrm{zu}$ wissen und die Therapie entsprechend anzupassen, um akute Blutzuckerentgleisungen zu vermeiden. In den hier vorgestellten Beiträgen finden sich viele weitere Beschreibungen von Subgruppen von Patienten, bei denen die Therapieempfehlungen und Therapieziele nur bruchstückhaft durch klinische Studien abgesichert sind (• Infobox 1).

\section{Dilemma bei der Implementierung von Leitlinien}

Wenn es um die Implementierung der evidenzbasierten Diabetesleitlinien geht, ergibt sich bei einer puristischen Interpretation das Dilemma, dass es für viele der Patienten kein durch eine evidenzbasierte Leitlinie vorgegebenes „Kochrezept" gibt, sondern oft nur noch die allgemeinen Behandlungsprinzipien als feste Säulen des Gebäudes, in dem sich ganz unterschiedliche Anwendungsszenarien abspielen. Dazu sollten wir uns an die wesentlichen Kriterien der evidenzbasierten Medizin nach Sackett et al. [2] erinnern, die auf folgenden 3 Säulen basiert:

1. wissenschaftliche Evidenz aus gut kontrollierten klinischen Studien,

2. medizinische Kenntnisse und der Erfahrung des Arztes sowie

3. Einbeziehung des Patienten in den Entscheidungsprozess entsprechend dem Willen und den Möglichkeiten des Betroffenen.

Daraus wird ersichtlich, dass die sog. individualisierte Medizin im Grunde die evidenzbasierte Medizin im Sinne Sacketts darstellt. 


\section{Lösung des Problems}

Das oben dargestellte Dilemma lässt sich nur auflösen, wenn der Arzt sehr gut ausgebildet und erfahren ist. Dazu muss er sich natürlich kontinuierlich fortbilden. Für die individuelle Behandlung seiner Patienten muss er aber auch jeweils herausfinden, was deren Wünsche sind, welche körperlichen und geistigen Fähigkeiten sie haben, in welchem sozialen Umfeld sie leben und inwieweit sie motiviert und dazu bereit ist, die medizinischen Prozeduren mitzutragen. Die Frage, ob es für alle Gruppen von Patienten speziell erprobte und bewährte Behandlungsansätze gibt, muss leider verneint werden. Zur Lösung dieses Problems müssten jeweils spezielle Studien mit sehr homogenen $\mathrm{Pa}$ tientengruppen aufgelegt werden, die jeweils eine ganz bestimmte Fragestellung bearbeiten, und selbst damit wiederum nur einen neuen Rahmen für die individualisierte Behandlung im Sinne der evidenzbasierten Therapie abstecken. Das ist ein weiter Weg, mit vielen noch anstehenden Aufgaben.

\section{Korrespondenzadresse}

\section{Prof. Dr. W.A. Scherbaum}

Klinik für Endokrinologie,

Diabetologie und Rheumatologie,

European Training Centre

in Endocrinology and Metabolism,

Universitätsklinikum Düsseldorf

Moorenstr. 5, 40225 Düsseldorf

scherbaum@uni-duesseldorf.de

Interessenkonflikt. Der korrespondierende Autor gibt an, dass kein Interessenkonflikt besteht.

\section{Literatur}

1. Adler Al, Stevens RJ, Manley SE et al (2003) Development and progression of nephropathy in type 2 diabetes: the United Kingdom Prospective Diabetes Study (UKPDS 64). Kidney Int 63:225-232

2. Sackett DL, Rosenberg WM, Gray JA et al (1996) Evidence based medicine: what it is and what it isn't. BMJ 312:71-72

3. Scherbaum WA, Landgraf R, Selbmann HK et al (2008). Diabetes-Leitlinien: gemeinsames Handeln aller Beteiligten. Dtsch Arztebl 105: A-1734-1736

4. Nationale VersorgungsLeitlinien, www.aezq.de

\section{Leserservice: Themenithersicht}
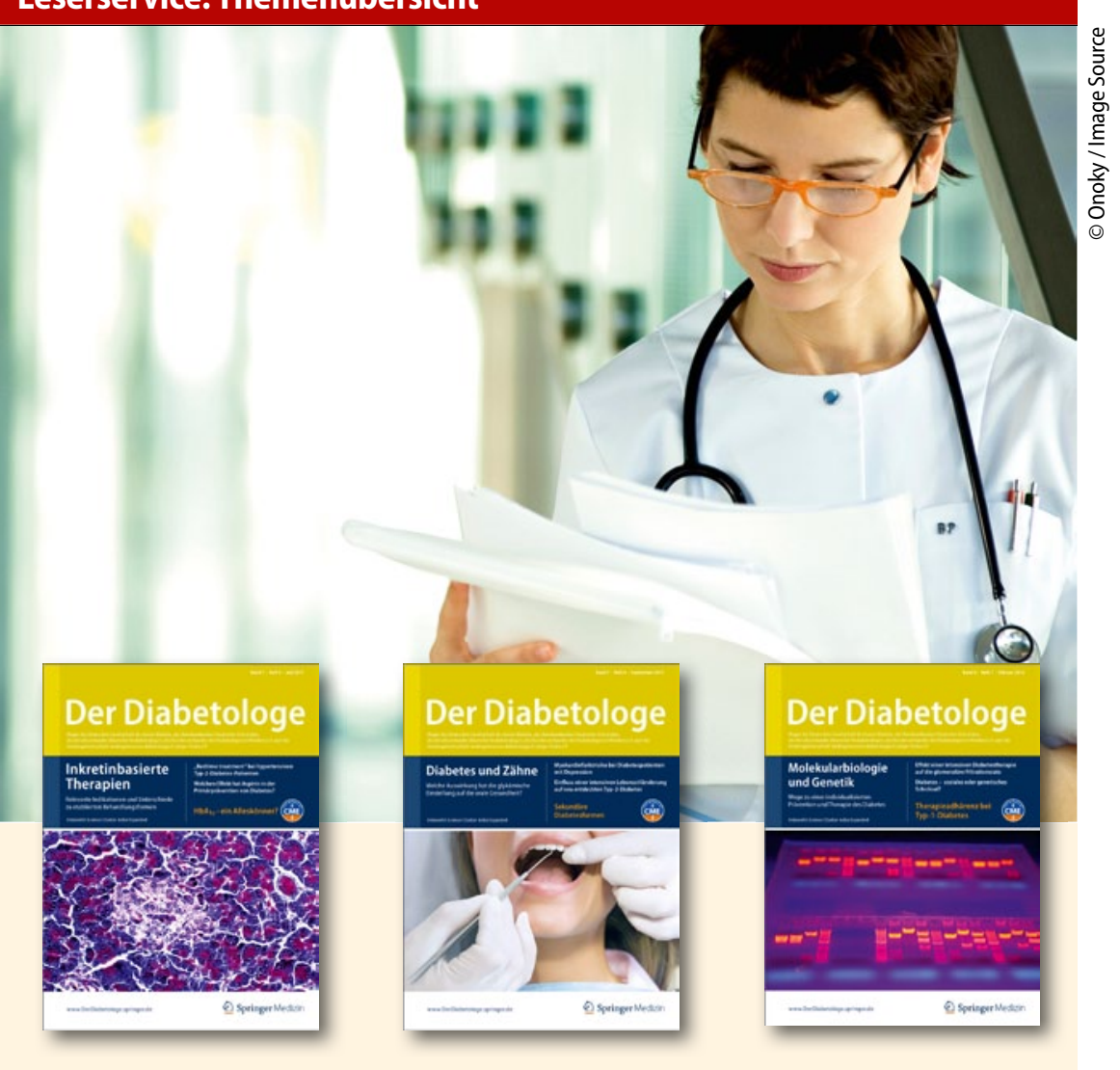

Der Diabetologe bietet Ihnen 8-mal pro Jahr umfassende und aktuelle Beiträge zu interessanten Themenschwerpunkten aus allen Bereichen der Diabetologie.

Möchten Sie ein bereits erschienenes Heft nachbestellen? Einzelne Ausgaben können Sie direkt bei unserem Kundenservice beziehen.
Jahrgang
2011
- 01/11 Sport
- 02/11 Gesundheitsökonomie
- 03/11 Update Diabetologie 2011
- 04/11 Diabetes und Herz
- 05/11 Inkretinbasierte Therapie
- 06/11 Diabetes und Zähne
- 07/11 Stationäre Diabetologie
- 08/11 Prävention bei Typ-1-Diabetes

\author{
Jahrgang \\ 2012 \\ - 01/12 Molekularbiologie und Genetik \\ - 02/12 Individuelle Therapieziele \\ in der Diabetologie \\ - 03/12 Praktische Diabetologie aus \\ Sicht der Diabetesberaterinnen \\ - 04/12 Telemedizin \\ - 05/12 Update \\ - 06/12 Diabetes und Krebs \\ - 07/12 Fettstoffwechselstörungen \\ - 08/12 Gestationsdiabetes
}

So erreichen Sie unseren Kundenservice:

Springer Customer Service Center GmbH Kundenservice Zeitschriften

Haberstr. 7

69126 Heidelberg

Tel.: +496221 345-4303

Fax: +49 6221 345-4229

E-Mail: leserservice@springer.com

\section{www.DerDiabetologe.springer.de}

